\title{
Experimental modular stand used for studies of the High Tibial Osteotomy
}

\author{
Ileana Ioana Cofaru ${ }^{1, *}$ \\ ${ }^{1}$ Lucian Blaga University of Sibiu, Computer Science and Electrical Engineering Department, 10, \\ 550025, Emil Cioran Street, No. 4, Sibiu, Romania
}

\begin{abstract}
The optimization of the surgery strategies for the correction of the axial deviations of the human lower member presents a great importance both for the affected patients and for surgeons. This article seeks to develop a modular stand used mainly for experimental study of the High Tibial Osteotomy (HTO). In the article it is also shown the relation between the axial deviations of the human leg and their corrections using HTO. The modular devices were designed, executed and used in the experimental research.
\end{abstract}

\section{Introduction}

In this paper we will achieve the design and 3D modelling for an experimental stand used for the study of the HTO. High Tibial Osteotomy (HTO) is a surgery strategy which treats the axial deviations of the human lower member. A correct alignment of the bones of the human lower member is defined by collinearity of the line segments which unite the centre of the hip joint, the centre of the knee articulation and the centre of the ankle.

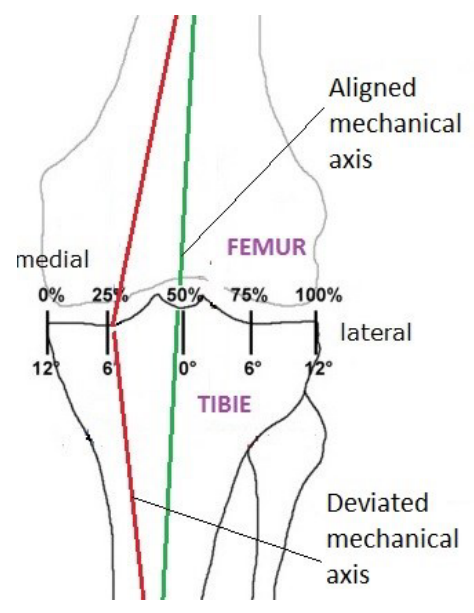

Fig. 1. Axial deviation of the mechanical axis in the knee articulation

\footnotetext{
* Corresponding author: cofaruioana@,gmail.com
} 
Any swerve from this axis becomes an axial deviation and it has important consequences on the health and the comfort of the patient affected by this deviations. In figure 1 it is shown both the knee articulation with the mechanical axis of the lower member both correctly aligned (green line) and one with the mechanical axis deviated (red line).

In the case where the mechanical axis is deviated we can observe that the mechanical axis of the tibia and of the femur aren't collinear, the axis of the leg appears broken and the loading does not take place in the centre of the knee articulations.

The main cause of this deviation is unicompartmental ghonartrosis, malady that unfortunately is more and more common today. The effect of this malady is the wear of the articular cartilage which leads to an incorrect loading of the knee and thus to the appearance of the deviations. From this moment there is a potentiality of the effects, meaning that as the deviation increases, the wear of the cartilage increases too and this usage involves a bigger deviation and so on.

One of the common procedures used in surgical practice for the correction of such axial deviations is the HIGH TIBIAL OSTEOTOMY (HTO). This procedure assume creation of the bone's wedge at the level of proximal human tibia in order to eliminate the axial deviations and to realign correctly the mechanical axis of the human lower member.
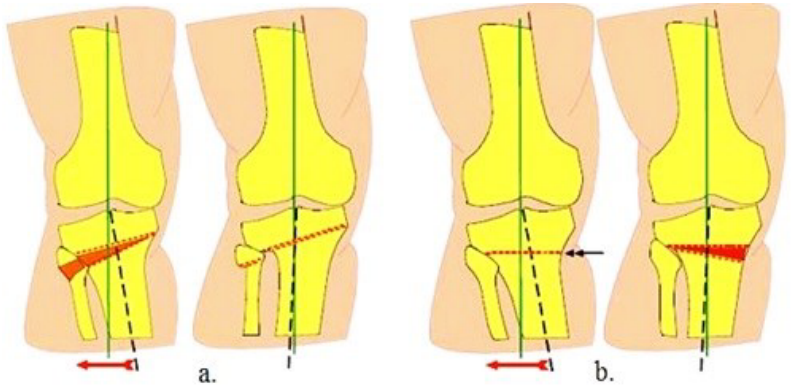

Fig. 2. High tibia osteotomy. $a$ - with closing wedge, $b$ - with opening wedge

In figure 2 it is shown a high tibial osteotomy and in it there are presented both the osteotomy with closing wedge and that with opening wedge $[2,4]$ (see Figure 2a). It can be seen that in case of osteotomy with closing wedge two cuttings are performed, the resulting wedge is eliminated and then the cuttings are placed one over the other (see Figure 2a) achieving in this way the axial correction of the tibia. In case of osteotomy with opening wedge only one cutting is performed and the wedge is created through the angular opening of the two resulted surfaces. Regarding the area in which the cuttings are performed in Figure $2 \mathrm{a}$ is an osteotomy from the lateral (exterior) side and in Figure 2.b is one from the medial (interior) side. It has to be underlined that the approach from the medial side, both in the case of closing and in the case of opening, doesn't require the cutting of the fibula which represent an important advantage.

This surgical procedures is very interesting also from a biomechanical point of view studying several problems such as: the good cutting of the bone material, the precision in achieving the angle of the wedge [3], the state of the stress and strain resulting in the area where the bone is not cut. In this area the bone will be rotated resulting axial alignment.

This variety of problems open up the possibility to achieve ample biomechanical studies regarding HTO. These studies can be: analytical, numerical, and experimental.

In this article we will present the design and modelling of the modular experimental stand destined for the experimental study of the HTO. 


\section{Requirements, necessities and functions of the stand}

The approach of the design of the experimental stand was achieved in a generalized manner. This was created modular in order to be used for the research of both HTO (closing wedge, opening wedge) and other orthopaedics surgeries.

For the design of the stand, specialty articles were studied in order to determine the current stage of research in this domain [5]. The other information came from analysing the necessities, from which we extracted the functions that the stand should accomplish. The constructive solutions were related to these functions.

The main objectives proposed for the development of the stand are:

- Establishing the needs and requirements that the stand should accomplish,

- The identification of the primary functions,

- The development of the implementation solutions,

- The 3D design of the stand

When it came to choosing the needs, a first step was the concretization of the current problems regarding the surgical strategies of osteotomy. Also, in order to establish the requirements, we took into account the opinions of surgeons, anaesthesiologists and last but not least of the patients.

By analysing all these aspects, the principal needs can be established. These are practically linked with the effects of specific surgeries. The stand must accomplish experimental studies regarding elements such as:

- the efficient planning before surgeries,

- enable the use of the knee functions as fast as possible,

- decreasing the stress states which appear in the tibia correction area,

- avoid further complications,

- equalise the distributions of the stress on the knee level,

- the bone fixation in correct position in order to achieve the correction of the mechanical axis,

- increase the safety of the surgery in order to prevent the developments of future infections.

Regarding requirements, at first we have to take into account the universality of the study, as it has to be applicable for every osteotomies type: opening, closing, medial, lateral, uniplanar or biplanar. Other important requirements are: achieving the fixation and orientation of the tibia and the possibility of closing and opening of the osteotomy wedge.

The constructive structure of the stand will be determined after the establishment and the analys is of the functions that the stand must carry out. These are:

- the orientations and fixations of the tibia both for closing and opening HTO,

- achieving the pushing in order to realize the closing of the osteotomy wedge,

- achieving the opening of the wedge for the opening osteotomy,

- the control of the contact surfaces position and of application of the forces in both situation from above;

- the orientation of the tibia-femur ensemble for experiments both for traction and compression stress,

- the possibility to adjust the position of the tibia and femur,

- achieving the controlled relative axial rotation between tibia and femur.

From the functions presented above, the last three are less important as far as osteotomies are concerned, but these were also took into account in order to confer a larger generalization of the stand. Also we considered for designing the stand the characteristics of the equipment and of the machines on which the stand will be assembled [6]. 


\section{Design of the stand. Elaborations of the implementation solutions.}

Starting from the functions shown in the last paragraph, we focused our attention on proposing the constructive solutions. Correspondence functions-solutions can be seen in the table 1.

Table 1. Functions and constructive solutions.

\begin{tabular}{|c|c|}
\hline Functions & Constructive solutions \\
\hline Tibia fixation & Spindle, Vice, Press, Clamp, Flange \\
\hline Femur fixation & Bar, Grip Clamp \\
\hline Push on tibia & Roll, Piercer \\
\hline $\begin{array}{c}\text { Angular positioning } \\
\text { Segmentation of the } \\
\text { tibia }\end{array}$ & „U” Frame \\
\hline
\end{tabular}

The criteria that helped with the evaluations of the optimal solutions have been: the cost, the functionality, and safety in exploitation of the parts of device. In order to determinate the optimal solutions the share calculus method was used [1], resulting in this way a structure of the dispositive which uses as rotation elements " $U$ " frames and for the orientation of the tibia the spindle. For pushing on the tibia in order to put together the bone segments (resulted after elimination of the wedge in the closing osteotomy) it was used a roll, and for creating an angular rotation of the bone segments after the elimination of the wedge in case of the closing osteotomy it was used a graduated wedge.

From the constructive point of view two big parts can be highlighted: an inferior ensemble responsible with the orientation and the fixation of the tibia and a superior ensemble responsible with the effective applications of the loadings.

The constructive solutions implemented will be shown in the following.

The inferior ensemble (see Figure 3) will accomplish the clamping and the positioning of the tibia. The tibia will be oriented and fixated in one shaft that permits a stabile fixation in three planes of the bone segments in a vertical plane related of the interior frame of the ensemble.

This ensemble consists form two shaped $U$ frames, one placed in the interior of the other one. The exterior frame is fix and the interior frame is mobile and can rotate around the articulations materialized by a pin placed at the level of the frame's lateral sides.

This structure permits the tilt and lock of the interior frame at various degrees, until $120^{\circ}$ in every way. The fixation of the assembly in a certain position is achieved through locking with two pins, which are inserted in the special holes situated on the lateral sides of the ensemble.

The shaft can be used with the tibia in the vertical position, horizontal position or tilted in any angle. 


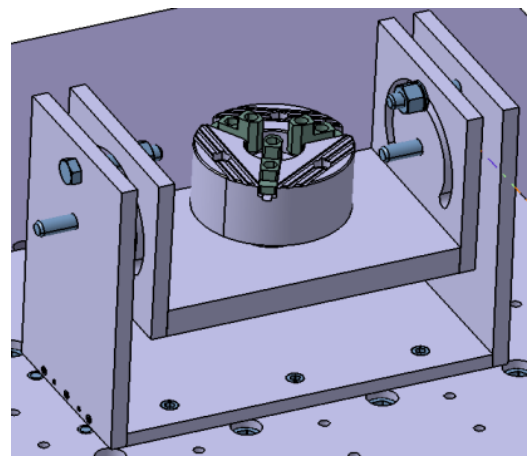

Fig. 3. High tibia osteotomy. $a$ - with closing wedge, $b$ - with opening wedge

The superior ensemble will differ depending of the experiments that are achieved. In principle two different modular devices will be made:

- for the study of the closing HTO,

- for the study of the opening HTO.

In figure 4 is shown an assembly view of the experimental stand used for the closing HTO. Apart from the inferior ensemble (described above) fixed on the table of the Instron universal tensile testing machine, we can also observe the superior ensemble fixed on the mobile traverse, situated in the superior area of the machine. The main functional part of the superior ensemble is the roll 1 which puts pressure on the superior side of the tibia in order to close the osteotomy wedge (see figure 4).

Roll 1 is articulated through a pin in fork 2, which is guided in the linked part 3, guidance that allows it to make anterior/posterior movements.

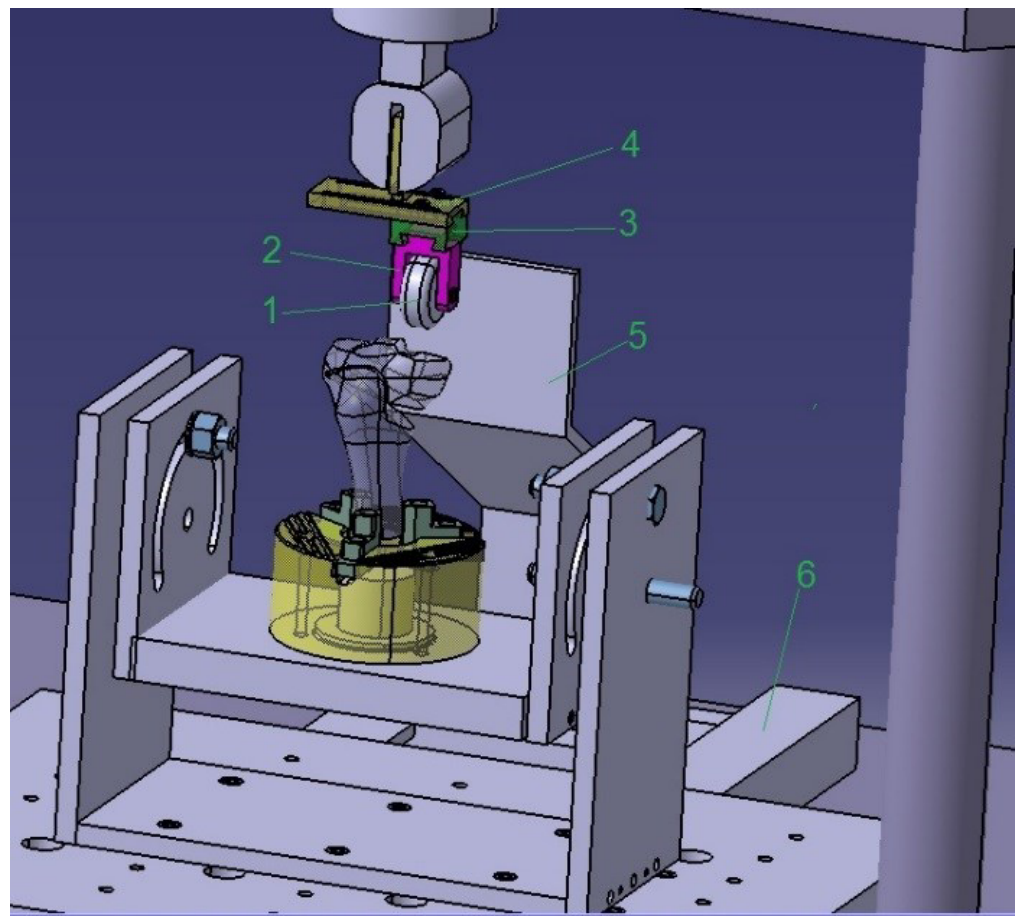

Fig. 4. Experimental stand used for studies of High Tibial Osteotomy with closing wedge 
For achieving left-right movements, part 3 is guided in the guiding plate 4 . On this plate there are a prismatic zone dimensioned in such a way that they can be assembled in the clamping devices of the machine. The two rectangular movements that the roll can accomplish are useful for determining both the point of forces application and mediallateral position for achieving the loading after HTO. The fixing in the needed positions will be realised through screwed assembly. The tibia was clamped in the shaft in vertical positions. In order to assure a correct position of the tibia in the device, it was designed a little ensemble which had the role to improve the guidance of the tibia in the clamping moment. This is useful in order to orientate the cutting in the transversal plane and the pressure exerted by the roll to be distributed evenly on the action area. For this auxiliary ensemble, we have proposed a plate vertically orientated 5 , which can approach or retreat from the $U$ frame moving on the guiding plate 6 . This ensemble will be mounted on the machine's table. The system is useful for the study of both the opening HTO and the closing HTO (see figures 4, 5).

The second experimental stand is destined for the studies of the opening HTO.

Analysing the assembly view (see figure 5), we can observe that the inferior ensemble used is the same as the inferior ensemble used in the previous stand. The difference is that the positioning of the ensemble isn't central with the symmetry centre situated in the axis machine but in the lateral part of the table's machine. Also the tibia that will be clamped in the shaft will not be fixed in the vertical position but close to the horizontal positions which allows the access of the graduated wedge 1 in the osteotomy area.

The superior ensemble is created from the same parts as is the closing HTO stand. The difference being that roll and fork were substituted in this case by the graduated wedge 1 guided by the linked part (see figure 5). The graduated wedge can execute movements both anterior-posterior and right-left.

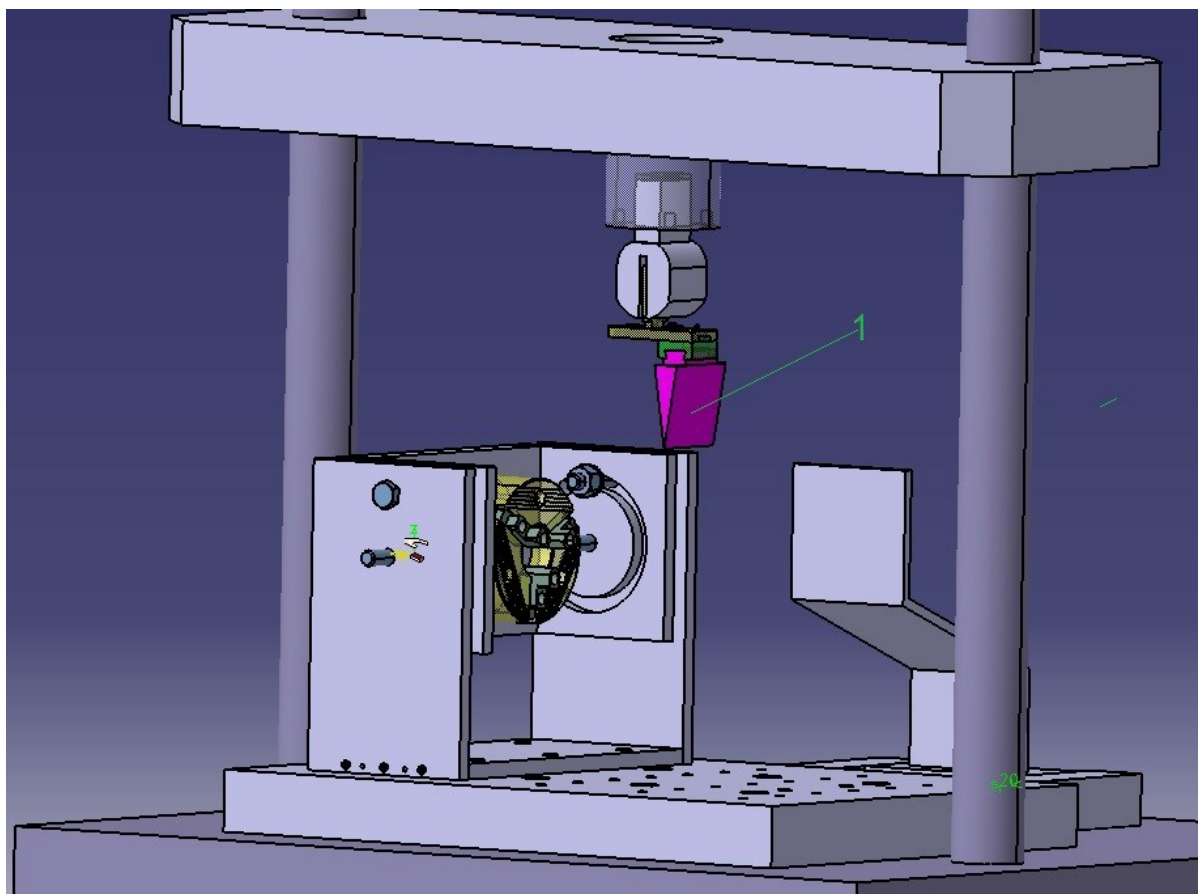

Fig. 5. Experimental stand used for studies of High Tibial Osteotomy with opening wedge 
An important element in this case is the possibility and necessity of the graduated wedge to rotate around of the axis of the mobile traverse of the machine. The machine ensures this function. For correct positioning of the tibia we will use the same guiding systemas the one presented at the previous stand

From a functional point of view the tibia is oriented and fixated in the shaft and it rotates around its own axis until the posterior surface meets the guiding plates. The next step is the orientation of the HTO cutting in the vertical plane in order to approach this with graduated wedge.

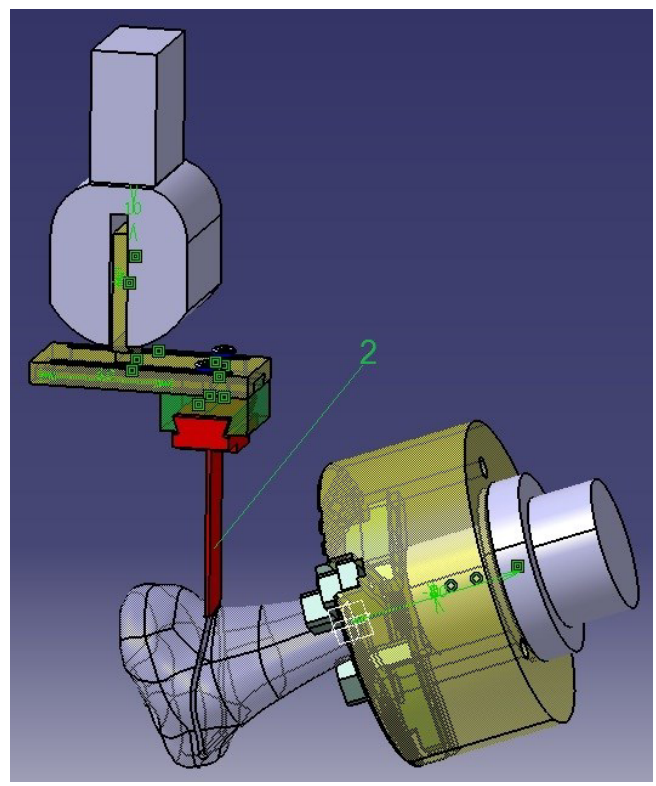

Fig. 6. The use of the searcher

For this purpose we will use the searcher 2 mounted in the superior ensemble instead of the graduated wedge (see figure 6). For moving the searcher in the HTO cutting, two translations on rectangular directions and one rotation in the horizontal plane are necessary. Also the interior frame together with the shaft and the tibia will perform a rotation until the searcher will be perfectly aligned in the HTO cutting (see figure 6).

This position will be conserved through the fixation screws form the $U$ frames in the angular slots. After the orientation was achieved, the searcher 2 is replaced with the graduated wedge (see figure 5). The graduated wedge was designed and realized starting from the real tool that is used in the surgical techniques. This has engraved on the lateral surfaces small channels with the lengths between 4 and $18 \mathrm{~mm}$. For achieving the required opening the graduated wedge is introduced in tibia until it touches the gradation corresponding to the needed angle.

Often in surgical practice it's made this approximation: $1 \mathrm{~mm}$ on the graduated wedge corresponds to a 1 degree angular correction.

For the both stands, the inferior ensemble and the superior ensemble are put in a cinematic chain through an extern machine. Concretely, for our experiments this machine will be an Instron universal tensile testing machine for traction, compression and buckling (Instron 5587, maximum force $300 \mathrm{kN}$ ) that can be found in our faculty. 


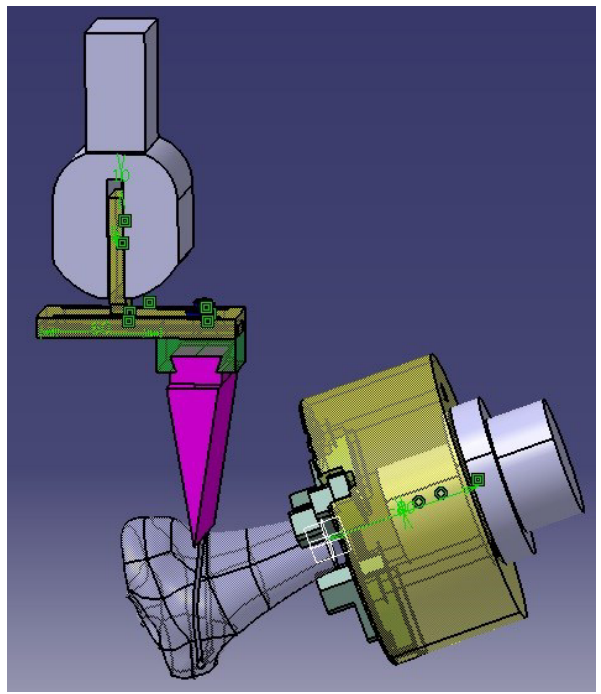

Fig. 7. The use of the graduated wedge

In figure 8, it is show the experimental stand destined for the study of the opening HTO, as this was developed and placed on the machine in our laboratory.

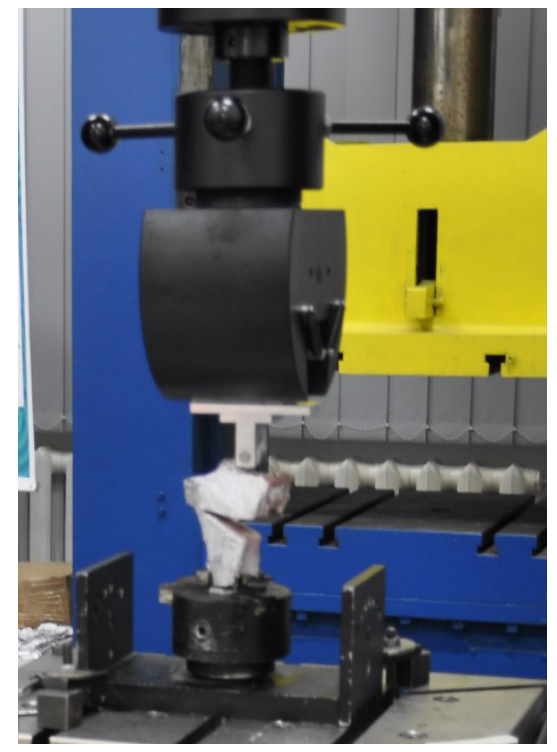

Fig. 8. Experimental stand used for studies of High Tibial Osteotomy with closing wedge

\section{Conclusions and further work}

In this paper, we have shown the designing of the devices used for the experimental study of the HTO and its variants. The designing was achieved starting from the necessity, requirements and functions of the stands. The modularization concept was used in order to cover a bigger area of research. 
These stands designed and developed will be an experimental platform of research for the PhD Students or teachers implied in the research concerning the optimization of the surgical procedures, in this case from the orthopaedic and traumatology fields.

The experiments achieved with these stands are very useful for the improvements of the quality of this type of surgeries.

\section{References}

1. I.I. Cofaru, - Researches regarding the biomechanics of the axial deviations of the human lower member and the development of the correspondent surgical devices, PhD Thesis, Sibiu, (2013)

2. AA. Amis, Knee Surg Sports Traumatol Arthrosc., 21(1), (2013)

3. D. Pape, K. Dueck, M. Haag, O. Lorbach, R. Seil, H. Madry A., Knee Surg Sports Traumatology Arthrosc., 21(1), (2013)

4. I.I. Cofaru, Annals of the University OfOradea, Romania, May, XXIII (2014)

5. I.I. Cofaru, P.D. Brindasu, N.F. Cofaru Applied Mechanics and Materials, 371 (2013)

6. M. Roman, R Fleacă, N. Cofaru, V. Oleksik, A. Pascu, C. Deac, I. Baier, The 11-th Congress of European Federation of National Association of Orthopaedics and Traumatology, Madrid, Spain, (2010) 